\title{
Abstract: An Empirical Examination of the Effectiveness of Different Types of Compensation in a Service Termination Context
}

\author{
Amin Nazifi and Dahlia El-Manstrly
}

\begin{abstract}
There is an increasing number of reports on termination of customer relationships initiated by major banks in the media. While there are some [short-term] benefits associated with termination of customer relationships such as improving profitability and increasing employees' productivity, our understanding of its negative consequences (negative WoM, compliant, retaliatory behaviors, etc.) and ways to address them is still very limited. Using a three dimensional perceived justice lens, this study looks at two distinct firm-initiated termination strategies (firm-oriented vs. customer-oriented) to compare their impact on customers' evaluations and behavioral reactions. A scenario-based experiment with a 2 (termination strategy: firm-oriented vs. customer-oriented) $\times 2$ (apology: present vs. absent) $\times 2$ (explanation: present vs. absent) $\times 3$ (monetary compensation: none vs. moderate vs. high) between-groups design is used. Based on the experiment conducted among 1248 US consumers in February 2016, we show that the two termination strategies significantly vary in terms of their perceived severity as well as their subsequent impacts on customers' attitudinal and behavioral reactions. In addition, we show that offering different types of compensation (both tangible and psychological) can be an effective strategy in reducing the negative consequences of firm-initiated service termination.
\end{abstract}

Keywords Service termination • Compensation - Perceived justice • Customers' negative behavioral reactions

References Available Upon Request.

A. Nazifi $(\bowtie) \bullet$ D. El-Manstrly

The University of Edinburgh, Edinburgh, UK

e-mail: amin.nazifi@ed.ac.uk; dahlia.el-manstrly@ed.ac.uk 